\title{
Normal Th1 development following long-term therapeutic blockade of CD154-CD40 in experimental autoimmune encephalomyelitis
}

\author{
Laurence M. Howard, ${ }^{1}$ Serge Ostrovidov, ${ }^{1}$ Cassandra E. Smith, ${ }^{1}$ Mauro C. Dal Canto, ${ }^{2}$ \\ and Stephen D. Miller ${ }^{1}$
}

${ }^{1}$ Department of Microbiology-Immunology and the Interdepartmental Immunobiology Center, and

${ }^{2}$ Department of Pathology, Northwestern University Medical School, Chicago, Illinois, USA

Address correspondence to: Stephen D. Miller, Department of Microbiology-Immunology,

Northwestern University Medical School, 303 East Chicago Avenue, Chicago, Illinois 60611, USA.

Phone: (312) 503-7674; Fax: (312) 503-1339; E-mail: s-d-miller@nwu.edu.

Received for publication October 9, 2001, and accepted in revised form November 19, 2001.

Experimental autoimmune encephalomyelitis (EAE) is a Th1-mediated demyelinating disease of the CNS with similarities to multiple sclerosis. We and others have shown that a short-term course of anti-CD154 mAb treatment to block CD154-CD40 interactions can be used to prevent or even treat ongoing PLP139-151-induced relapsing EAE. However, little is known of the long-term effects of CD154 blockade on the development of antigen-specific $T$ cell function. Here, we show that shortterm treatment with anti-CD154 at the time of PLP139-151/CFA immunization inhibits clinical disease for up to 100 days after immunization. At this point, comparable numbers of Th1 cells are observed in anti-CD154 and control Ig-treated mice, as assessed by antigen-specific ELISPOT assays. Thus, the long-term $\mathrm{Th} 1 / \mathrm{Th} 2$ balance is largely unaffected. Inflammatory responses are diminished in anti-CD154-treated mice, as indicated by reduced in vivo delayed-type hypersensitivity and reduced levels of splenic IFN- $\gamma$ secretion in vitro. However, upon adoptive transfer of T cells isolated from the spleens of anti-CD154-treated mice, these cells contributed as effectively to clinical disease as those obtained from control-treated mice. Thus, anti-CD154 therapy leads to long-term therapeutic efficacy without exerting a long-term influence on Th1 development.

J. Clin. Invest. 109:233-241 (2002). DOI:10.1172/JCI200214374.

\section{Introduction}

Experimental autoimmune encephalomyelitis (EAE) is a CD4 Th1-mediated autoimmune CNS demyelinating disease with clinical and pathologic similarity to the human disease multiple sclerosis (MS) (1). Immunization of SJL mice with myelin proteins or their encephalitogenic peptides in CFA leads to the development of relapsing EAE characterized by moderate to severe hind limb paralysis during the acute disease episode, followed by remission and then spontaneous disease relapse (2). While the etiology of MS is unclear, epidemiologic evidence suggests a possible infectious origin $(3,4)$, which may lead to autoimmunity by a variety of potential mechanisms (5). Current therapeutic strategies for MS are of limited efficacy and complicated by the complex nature of the disease immunopathology.

Blockade of the CD154-CD40 ligand pair interaction is an immunoregulatory strategy that has been used successfully in numerous autoimmune disease models including EAE (6-12). CD40 is constitutively expressed by dendritic cells, B cells, monocytes/macrophages, and other immune cells as well as some endothelial and specialized cells (13). The ligand for CD40, CD154, has a more restricted expression pattern that is transiently upregulated on activated T cells (14). The CD154CD40 ligand pair plays a central role in the develop- ment of the immune response (14) and in T cell effector function $(15,16)$ and is critical to sustaining the immune response (17-20). Specifically, during Th cell differentiation, CD40 ligation has been linked with the upregulation of IL-12, which itself may result in Th1 skewing $(21,22)$. We previously showed that antiCD154 therapy is effective in both preventing EAE and treating ongoing disease (8), not by inducing Th2 skewing, but rather by delaying the peak and diminishing the amplitude of the myelin peptide-specific Th1 response (16). We proposed that the eventual development of Th1 cells might be due to CD40-independent routes of IL-12 induction (23). Both B cell and dendritic cell survival and maturation are dependent upon CD40 ligation $(20,21)$, resulting in the diminished or absent immune responses observed in CD40 knockout mice (21). Finally, it has been shown that CD40 ligation is critical for effector functions of both CD4 and CD8 $T$ cells $(15,16,21,24)$.

Currently, the long-term effects of CD154 blockade are unknown. While we demonstrated that CD154 blockade can inhibit both initiation of EAE and ongoing disease over a short period of time (8), the longterm effectiveness of this therapy on clinical disease and on the development of antigen-specific immune responses is not known. Furthermore, little is known 
about the functionality of Th1 cells that differentiate under this therapeutic strategy: are these cells functionally different and less able to contribute to clinical disease at some future stage? In this paper, we examine the long-term efficacy of CD154 blockade in preventing the induction of EAE. We show that short-term treatment with anti-CD154 at the time of PLP139-151/CFA immunization was effective at inhibiting clinical disease for up to 100 days after immunization despite the fact that peripheral myelin peptide-specific Th1 responses were comparable in anti-CD154 and control antibody-treated mice. Additionally, and most importantly with respect to therapeutic strategies involving CD154 blockade, peripheral $\mathrm{T}$ cells in antibody-treated mice were fully capable of contributing to clinical disease in a cotransfer model, suggesting that their functional capacity to differentiate into effector Th1 cells was not affected.

\section{Methods}

Mice. Five- to six-week-old female SJL mice were obtained from Harlan Sprague Dawley Inc. (Indianapolis, Indiana, USA). Mice were housed under barrier conditions in the Center for Comparative Medicine at Northwestern University Medical School. Paralyzed mice were afforded easier access to food and water. DO11.10 congenic mice were bred within the facility and BALB/cAnNCr mice were obtained from NCI Laboratories (Frederick, Maryland, USA).

Peptides. PLP139-151 (HSLGKWLGHPDKF), PLP178-191 (NTWTTCQSIAFPSK), and OVA323-339 (ISQAVHAAHAEINEAGR) were purchased from Peptides International Inc. (Louisville, Kentucky, USA). Purities (>97\%) were confirmed by mass spectroscopy. Induction of EAE, immunization, and treatments. Active disease was induced by subcutaneous immunization with $100 \mu$ lof incomplete Freund's adjuvant containing 400 $\mu \mathrm{g} / \mathrm{ml}$ of Mycobacterium tuberculosis H37Ra (Difco Laboratories, Detroit, Michigan, USA) divided between the hind flanks and upper dorsal region. For in vivo activation of DO11.10 T cell receptor (TCR) transgenic T cells, $5 \times 10^{6} \mathrm{CD} 4$ magnetic bead-purified $\mathrm{T}$ cells from a DO11.10 mouse spleen were transferred intraperitoneally 3 days prior to immunization. For EAE adoptive transfer experiments, draining lymph node cells were obtained 10 days after immunization and cultured at $8 \times 10^{6}$ cells per milliliter in the presence of $25 \mu \mathrm{M}$ PLP139-151 peptide for 4 days. The blasts obtained from these cultures were transferred intraperitoneally at $2 \times 10^{6}$ blasts per naive recipient mouse. T cells were obtained from spleens of either control or anti-CD154-treated mice 30 days after immunization, and magnetic bead-purified using anti-CD90.2-coated beads, as per the manufacturer's instructions. We transferred $5 \times 10^{6} \mathrm{~T}$ cells intraperitoneally to naive recipient mice either alone or in combination with lymph node blasts obtained as above. For OVA323-339 peptide priming of BALB/cAnNCr mice, the same protocol was used with $500 \mu \mathrm{g} / \mathrm{ml} \mathrm{OVA323-339}$ peptide dosed at $100 \mu \mathrm{l}$ per animal.
Purified anti-CD154 (MR-1) and hamster IgG were purchased from Bioexpress Cell Culture Services (Lebanon, New Hampshire, USA) and Caltag Laboratories Inc. (Burlingame, California, USA), respectively. SJL mice were treated four times with $200 \mu \mathrm{g}$ of control hamster Ig or MR-1 antibody intraperitoneally in a total volume of $500 \mu \mathrm{l}$ every other day starting at the time of immunization. BALB/cAnNCr mice were treated intraperitoneally 1 day prior to OVA323-339 immunization and each day after priming until day 3 with 200 $\mu \mathrm{g}$ of control hamster Ig or MR-1 antibody.

Clinical evaluation. Mice in treatment groups were evaluated daily for clinical signs of disease for up to 105 days after inoculation. Clinical severity was assessed on a 0-to-5 scale as follows: grade 1, limp tail; grade 2, limp tail and hind limb weakness (waddling gait); grade 3 , partial hind limb paralysis; grade 4, complete hind limb paralysis; and grade 5, moribund. The data are plotted as the daily mean clinical score for all animals in a particular treatment group.

Serological evaluation of antigen-specific Ig production. Serum was obtained 25 days after immunization. ELISA was used for detection of antigen-specific antibody responses to PLP139-151. Briefly, Maxisorb plates (NUNC A/S, Roskilde, Denmark) were coated overnight at room temperature with $100 \mu$ l of PLP139-151 peptide at $5 \mu \mathrm{g} / \mathrm{ml}$. Plates were washed and blocked with PBS containing 5\% BSA and 10\% normal goat serum. One hundred milliliters of $1: 100$ to $1: 10,000$ serial serum dilutions were then added to multiple replicate wells and incubated at room temperature for 1 hour and washed, and horseradish peroxidase-conjugated anti-mouse antibody (Caltag Laboratories Inc.) was then added. Wells were then washed and substrate solution added (tetramethyl benzidine substrate; BioFX Laboratories Inc., Owing Mills, Maryland, USA). The plates were read at $450 \mathrm{~nm}$ on a microtiter plate reader (Molecular Devices Corp., Sunnyvale, California, USA).

Delayed-type bypersensitivity responses. Delayed-type hypersensitivity (DTH) responses to PLP139-151 were quantitated using a 24-hour ear swelling assay. Prechallenge ear thickness was determined using a Mitutoyo model 7326 engineer's micrometer (Schlessinger's Tools Inc., Brooklyn, New York, USA). DTH responses were elicited by injecting $10 \mu \mathrm{g}$ of peptide (in $10 \mu \mathrm{l}$ of saline) into the dorsal surface of the ear using a 100- $\mu \mathrm{l}$ Hamilton syringe (Hamilton Co., Reno, Nevada, USA) fitted with a 30-gauge needle. Twenty-four hours after ear challenge, the increase in ear thickness over prechallenge measurements was determined. Results are expressed in units of $10^{-4}$ in. \pm SEM.

Histology. Anesthetized mice were sacrificed by total body perfusion with chilled $3 \%$ glutaraldehyde in PBS, $\mathrm{pH}$ 7.3. Spinal cords were removed by dissection, cut into 1-mm-thick coronal segments, post-fixed in 1\% osmium tetroxide, dehydrated, and embedded in Epon as previously described (25). Toluidine blue-stained sections from ten segments per mouse were read blindly and scored as follows: $+/-$, mild inflammation with- 
out demyelination; 1+, inflammation with focal demyelination; $2+$, inflammation with multiple foci of demyelination; $3^{+}$, marked inflammation with bilateral, converging areas of demyelination; $4+$, extensive bilateral areas of demyelination and remyelination.

$T$ cell proliferation assays. Spleen and lymph node cells were obtained from mice at various stages of disease progression and dissociated cells were cultured in 96-well microtiter plates (Costar tissue culture; Corning Inc. Life Sciences, Acton, Massachusetts, USA) at a density of $5 \times 10^{5}$ viable cells per well as described previously (9). Cultures were pulsed with $1 \mu \mathrm{Ci}$ of ${ }^{3} \mathrm{H}-\mathrm{TdR}$ (ICN Radiochemicals Inc., Irvine, California, USA) after 72 hours and harvested at 96 hours, and ${ }^{3} \mathrm{H}-\mathrm{TdR}$ uptake was detected using a Packard Topcount microplate scintillation counter (Packard Instrument Co., Meriden, Connecticut, USA). Results are expressed as mean of triplicate cultures \pm SEM. Supernatants were harvested on days 1-4 and analyzed for IL-2, IL-4, IL-5, and IFN- $\gamma$ by capture ELISA (Endogen Inc., Woburn, Massachusetts, USA) according to the manufacturer's protocol.

ELISPOT assays. Spleen and lymph node cells were obtained from mice cultured in 96-well microtiter ELISPOT plates (Whatman Polyfiltronics, Clifton, New Jersey, USA) that had been coated overnight with capture antibodies to IL-2, IL-5, or IFN- $\gamma$ (BD Pharmingen, Mountain View, California, USA). Total cell numbers recovered were determined by use of a hemocytometer. ELISPOT cultures were carried out as previously described (17). ELISPOTs were counted using an ELISPOT plate reader and software (Cellular Technologies Inc., Cleveland, Ohio, USA). At least four wells per sample were counted, and presented as a mean value.

Flow cytometric analysis. Three days after immunization of BALB/cAnNCr recipients of transferred DO11.10 cells, lymph node cells were harvested and analyzed for expression of various homing receptors by flow cytometry. Clonotypic KJ1-26 antibody and CD45 expression (BD Biosciences Pharmingen, San Diego, California, USA) identified DO11.10 TCR transgenic T cells. Cells were stained for expression of CD44, CD49d, and CD62L using antibodies, and for P-selectin ligand expression using a human IgG-P-selectin chimeric protein obtained from $\mathrm{BD}$ Pharmingen. In addition, E-selectin-Ig (a kind gift from Geoffrey Kansas, Northwestern University Medical School) chimeric protein was used to identify functional E-selectin ligand expression. Binding to cells was identified using secondary phycoerythrin- (PE-) or FITC-conjugated antibodies to human IgM and IgG, respectively (Jackson ImmunoResearch Laboratories Inc., West Grove, Pennsylvania, USA). Samples were run on a Becton Dickinson FACSCalibur flow cytometer, with a minimum of 5,000 DO11.10 transgenic T cells counted.

Statistical analyses. Comparison of the percentage of animals with clinical disease between any two groups of mice was analyzed using Fisher's exact test on Instat software (Graphpad Software Inc., San Diego, California, USA).

\section{Results}

Short-term CD154 blockade results in long-term inbibition of EAE induction. Short-term blockade of CD154-CD40 interactions effectively inhibits the induction of EAE for up to 40 days after immunization $(6,8,26,27)$. More recently, we have shown that anti-CD154-induced protection is not the result of a Th1-to-Th2 switch; rather, blockade delays the peak and diminishes the magnitude of the peripheral myelin peptide-specific Th1 response and downregulates T cell effector function in the CNS (16).

Since T cells with encephalitogenic potential continue to be induced in the periphery of SJL mice treated with anti-CD154 at disease induction, yet no clinical disease is observed over the first 40 days, we examined the long-term capacity of CD154 blockade to inhibit disease development. Treatment with MR-1 at time of immunization resulted in the long-term inhibition of clinical disease induction (Figure 1a). Control-treated mice demonstrated $100 \%$ disease incidence within the first 20 days, while only 2 of 16 mice (12.5\%) treated with MR-1 antibody developed mild, short-lived clinical signs of disease, after the initial 40 days. Long-term inhibition of clinical disease correlated with the histopathology scores from spinal cord sections taken from representative mice 100 days after cessation of treatment (106 days after immunization) (Figure 1, b and c; Table 1), where very few immune cell infiltrates and no demyelination was observed in MR-1- compared with control antibody-treated mice. Thus, shortterm treatment of mice from the time of immunization resulted in nearly complete inhibition of EAE.

Anti-CD154 treatment inhibits in vivo PLP139-151-specific antibody and DTH responses. While CD154 blockade inhibited induction of EAE, we wanted to determine how effective the antibody treatment was at blocking CD40 ligation in vivo. IgG isotype switching is CD40-dependent, as demonstrated in human hyper-IgM syndrome and also in the murine CD40 or CD154 knockouts, where little or no antigen-specific IgG isotypes can be produced (28-30). As shown in Figure 2a, the PLP139-151-specific serum IgG antibody response is dramatically reduced at 25 days after priming, but not completely ablated by CD154 blockade at the doses used.

We have previously shown that DTH responses to PLP139-151 were inhibited for up to 25 days after anti-CD154 treatment (8). To determine whether this inhibition was maintained, DTH responses were determined in mice 100 days after priming. As shown in Figure 2b, PLP139-151-specific DTH responses were still significantly reduced nearly 90 days after the end of the antibody treatment. However, the DTH was still significantly above background levels in naive mice, reaching approximately two-thirds that of control antibody-treated mice.

Effects of short-term anti-CD154 therapy on in vitro peptidespecific proliferative and cytokine responses. We next evaluated the levels of PLP139-151-specific proliferation and cytokine responses in the lymph nodes and spleens of mice treated with anti-CD154 mAb. No significant 


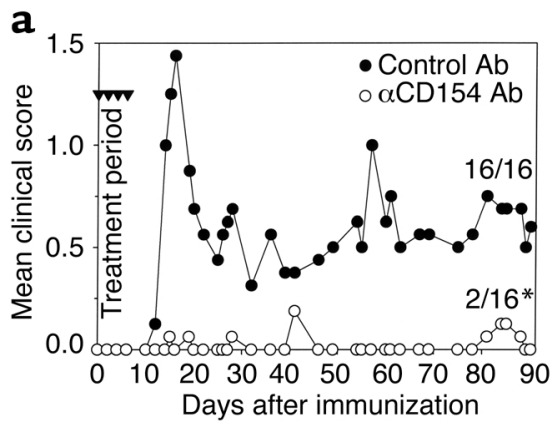

b



C

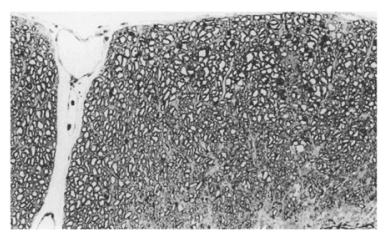

\section{Figure 1}

Short-term anti-CD154 mAb therapy causes long-term inhibition of EAE induction. (a) The indicated numbers of SJL mice were immunized with PLP139-151/CFA on day 0 and treated with $200 \mu \mathrm{g}$ of either hamster IgG or anti-CD154 antibody (MR-1). Data represent combined results obtained from two separate experiments. ${ }^{*}$ Disease incidence was significantly less $(P<0.0001)$ in anti-CD154-treated mice. (b and $\mathbf{c})$ Mice immunized and treated with control $\lg (\mathbf{b})$ or anti-CD154 (c) were sacrificed 105 days after immunization and sections were taken from lumbar and thoracic spinal cord sections, as described in Table 1. Sections demonstrate the continued normal appearance of spinal cord from anti-CD154-treated versus extensive immune cell infiltration, demyelination, and scarring observed in control Ig-treated mice.

differences in the levels of peptide-specific proliferation were observed in either the spleen or the draining lymph nodes of anti-CD154- versus control Ig-treated mice 100 days after immunization (Figure 3a). Additionally, no significant differences in cell numbers in the lymph nodes or spleens of these mice were observed (data not shown). Cytokine secretion profiles were determined from supernatants of PLP139-151-stimulated lymph node and splenic $T$ cells, and no detectable peptide-specific IL-4 or IL-5 secretion was observed (data not shown). In contrast, equivalent high levels of IL-2 were produced by both lymph node and splenic T cells of antiCD154- and control Ig-treated animals (Figure 3b). Similarly, IFN- $\gamma$ secretion by lymph node $\mathrm{T}$ cells from both anti-CD154 and control-Ig treated mice was identical 100 days after immunization (Figure 3b). However, IFN- $\gamma$ secretion from splenic $T$ cells was reduced by about $40 \%$ in anti-CD154- compared with control-treated animals (Figure $3 b$ ). The reduced PLP139-151 specific IFN- $\gamma$ secretion observed in the spleens of antiCD154-treated mice may be due to either reduced numbers of IFN- $\gamma$-secreting cells or their reduced levels of secretion. To address this, ELISPOT was used to determine the frequencies of Th0, Th1, and Th 2 cells in the lymph node and splenic compartments (Figure 4). Consistent with the results of the proliferation and IL-2 secretion assays, no significant difference in the frequencies of IL-2-secreting cells was observed in the draining lymph node or splenic compartments. This was also true for the frequencies of IL-5-secreting cells, although very few IL-5-secreting cells were identified in either group at this late point. In contrast to the reduced IFN- $\gamma$ levels secreted by splenic T cells as determined by ELISA (Figure $3 \mathrm{~b}$ ), there was no significant difference in the frequencies of IFN- $\boldsymbol{\gamma}$-secreting cells from
anti-CD154- versus control-treated mice. This suggests that comparable numbers of Th1 cells appear in the lymph node and splenic compartments in anti-CD154and control-treated mice. The levels of IFN- $\gamma$ production by $\mathrm{T}$ cells from the splenic compartments of anti-CD154 versus control-treated mice, on a per-cell basis, are marginally reduced. However, these cells can and do secrete significant amounts of Th1 cytokines such as IFN- $\gamma$.

CD154 blockade does not in bibit the early expression of homing receptors on antigen-specific $T$ cells. Recently, we showed that $\mathrm{CD} 154$ blockade resulted in the inhibition of $\mathrm{T}$ cell effector function in the CNS of recipients of encephalitogenic $\mathrm{T}$ cell transfers by inhibiting the expansion and/or retention of the effector cells in the target organ (16). We thus examined the effect of short-term antibody therapy on early antigen-induced expression of homing receptors involved in $\mathrm{T}$ cell trafficking to the CNS. $\mathrm{BALB} / \mathrm{cAnNCr}$ mice were treated intraperitoneally with $200 \mu \mathrm{g}$ of control Ig or anti-CD154 every day over a 4-day period. On day $+1,5 \times 10^{6}$ naive OVA323-339-specific DO11.10 $\mathrm{T}$ cells were transferred to each treatment
Table 1

Summary of histology of spinal cord sections from control- and anti-CD154-treated mice, 100 days after treatment and immunization

\begin{tabular}{|c|c|c|c|c|}
\hline \multirow[b]{2}{*}{$\begin{array}{l}\text { Experiment } \\
\text { number }^{A}\end{array}$} & \multicolumn{2}{|c|}{ Hamster Ig controls } & \multicolumn{2}{|c|}{ Anti-CD154-treated } \\
\hline & $\begin{array}{l}\text { Disease score } \\
\text { (acute/relapse) }\end{array}$ & $\begin{array}{c}\text { Histopathology } \\
\text { score }^{\mathrm{C}}\end{array}$ & $\begin{array}{c}\text { Disease score } \\
\text { (acute/relapse) }\end{array}$ & $\begin{array}{c}\text { Histopathology } \\
\text { score }\end{array}$ \\
\hline $1 \mathrm{a}$ & $3 / 2$ & ++ & $0 / 0$ & 0 \\
\hline $1 b$ & $4 / 3$ & +++ & $0 / 0$ & 0 \\
\hline $2 \mathrm{a}$ & $2 / 1$ & + & $0 / 0$ & 0 \\
\hline $2 b$ & $2 / 2$ & + & $0 / 0$ & 0 \\
\hline
\end{tabular}

ALumbar spinal cord sections from mice treated at the time of immunization for four treatments every other day with either hamster Ig control or anti-CD154 antibody were assessed for CNS histopathological changes 100 days after the end of treatment as detailed in Methods. A total of four mice taken from two separate experiments were used as shown. BPeak disease score from acute and relapsing phases of disease for the individual mice examined in each experiment. CTen sections were examined for each mouse and scored for disease on the following scale: 0 , no disease; +/-, meningitis; +, focal infiltration and demyelination; ++, multiple infiltrates and demyelination; +++, confluent infiltrates and demyelination. 
a

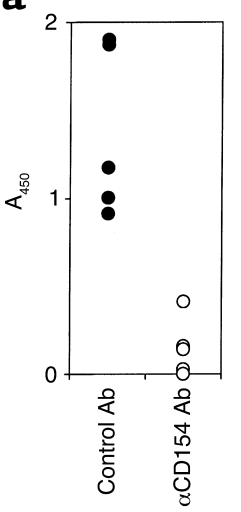

b

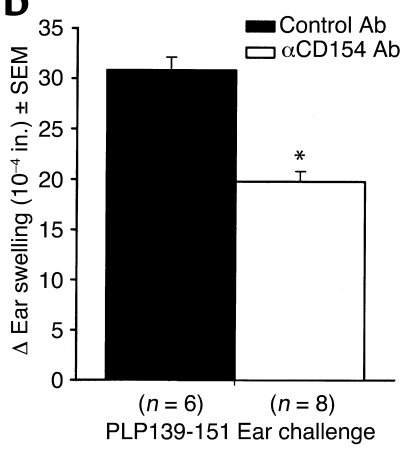

\section{Figure 2}

Short-term anti-CD154 therapy inhibits in vivo PLP139-151-specific antibody and DTH responses. Mice were immunized with PLP139-151/CFA and treated with control Ig or anti-CD154 as described in Figure 1. (a) Sera obtained from mice 25 days after immunization were analyzed for anti-PLP139-151-specific IgG levels. Data were obtained from five serological samples combined from two separate experiments. (b) PLP139-151-specific DTH responses were measured at 100 days after immunization. Data presented are the combined results from two separate experiments, and the number of mice in each group is marked below each bar. ${ }^{*} \mathrm{DTH}$ response was significantly less $(P<0.05)$ in anti-CD154-treated mice. group and the mice were immunized subcutaneously with $500 \mu \mathrm{g}$ of OVA323-339/CFA. On day +3 , the expression of various homing receptors was assayed on OVA323-339-specific $\mathrm{T}$ cells in the draining lymph nodes. As shown in Figure 5, the expression levels and percentages of $\mathrm{T}$ cells expressing homing receptors important for trafficking of $\mathrm{T}$ cells to inflammatory sites were comparable on DO11.10 T cells from mice treated with anti-CD154 or control antibody. This was true for homing receptors critical for $\mathrm{T}$ cell entry into the CNS, i.e., CD44 and CD49d (very late antigen-4 [VLA-4]) (Figure 5 , $a$ and $b$ ), and for other homing receptors including CD62L and E- and P-selectin ligands (Figure 5, c-e). Since E- and P-selectin ligands are dependent upon IL-12 secretion by antigen-presenting cells (APCs) during $\mathrm{T}$ cell differentiation, it appears likely that IL-12 secretion is largely unaffected by anti-CD154 treatment.

$T$ cells from asymptomatic anti-CD154-treated mice have encephalitogenic potential. It appears that significant numbers of self-reactive PLP139-151-specific Th1 cells circulate throughout the mouse immune system in antiCD154-treated mice in numbers comparable to those of hamster Ig-treated controls. However, clinical disease is not readily observed in anti-CD154-treated animals, even 90-100 days after immunization, when the mAb will in all likelihood have been cleared. To determine whether these peripheral PLP139-151-specific Th1 cells had the ability to contribute to clinical EAE, splenic T cells were isolated from anti-CD154- and control-treated mice 30 days after immunization. These cells were then transferred to naive recipient mice with or without

\section{Figure 3}

Short-term anti-CD154 therapy has no long-term effect on specific proliferative responses. (a) Splenic and lymph node (LN) cells were obtained 100 days after immunization, and in vitro PLP139-151-specific proliferative responses were determined by ${ }^{3} \mathrm{H}-\mathrm{TdR}$ incorporation. Data are representative of two separate experiments. (b) Supernatants from proliferative cultures described above were analyzed for secretion of IL-2, IL-4, IL-5, and IFN- $\gamma$ by capture ELISA. IFN- $\gamma$ secretion from splenic $T$ cells was significantly reduced in anti-CD154-treated mice. Data are representative of two separate experiments with three mice per experimental group. There was no detectable IL-4 or IL-5 secretion observed from any culture supernatants. addition of encephalitogenic Th1 cells obtained from the draining lymph nodes of mice 10 days after PLP139-151/CFA immunization and activated for 4 days in vitro with PLP139-151. As expected, in vitro-activated PLP139-151-specific T cell blasts could transfer clinical EAE to naive SJL recipients (Figure 6). Cotransfer of splenic $\mathrm{CD}_{4}{ }^{+} \mathrm{T}$ cells from either anti-CD154-treated or control Ig-treated mice, with peptide-specific $\mathrm{T}$ cell blasts, caused a significant worsening of clinical disease in recipient mice. In other experiments, the cotransfer of

a

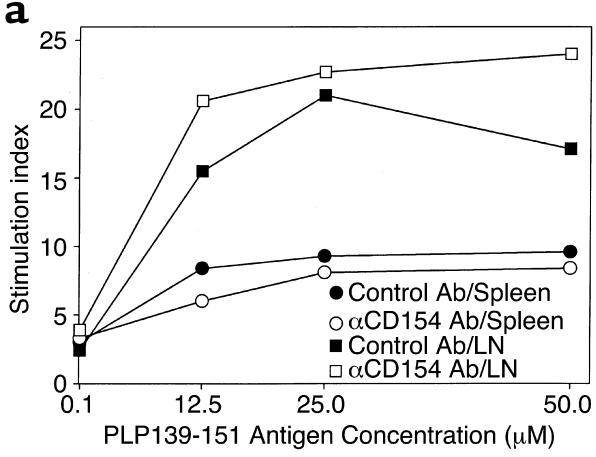

b
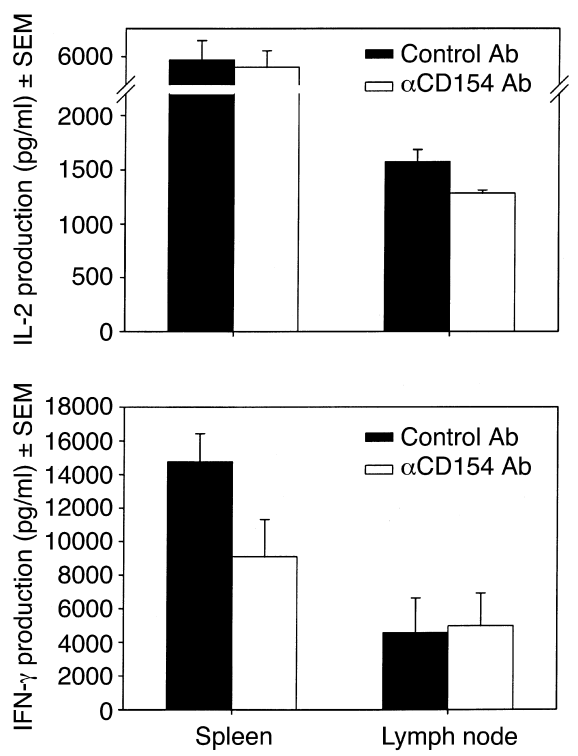


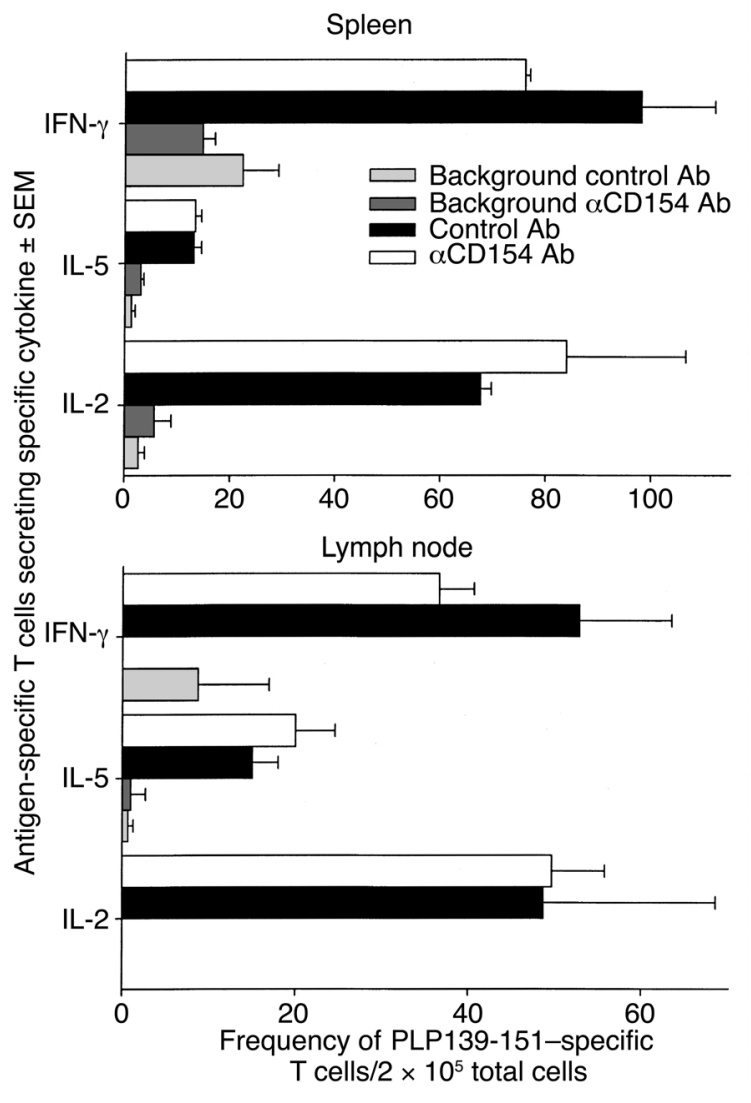

$\mathrm{CD}^{+} \mathrm{T}$ cells from the spleens of mice immunized with an irrelevant viral peptide from Theiler's virus (VP2 70-86) or OVA323-339 peptide did not modulate disease (data not shown). This indicates that peripheral $\mathrm{T}$ cells from asymptomatic anti-CD154-treated mice were fully capable of contributing to clinical EAE (Figure 6). However, neither splenic population was capable of initiating development of clinical disease de novo in the recipients when given alone.

\section{Discussion}

CD40 ligation has been shown to play a central role in the induction and pathogenesis of many experimental diseases, and blockade of this interaction has shown great

\section{Figure 5}

Short-term anti-CD154 therapy does not affect the early expression of homing receptors on OVA323-339-specific transgenic T cells in the draining lymph node. Five million DO11.10 transgenic OVA323-339-specific $T$ cells were transferred to naive BALB/c recipients, and the recipients were immunized with $500 \mu \mathrm{g}$ of OVA323-339/CFA and treated with either control Ig or anti-CD154 as described in Methods. Three days after immunization, lymph node cells were obtained and flow cytometric analysis of homing receptors evaluated ex vivo on $\mathrm{KJ} 1-26$ cells. Antigens critical for T cell entry into the CNS (CD44 and CD49d; $\mathbf{a}$ and b) and into the lymph node (CD62L; c), and IL-12-dependent homing receptors (E- and P-selectin ligands; $\mathbf{d}$ and $\mathbf{e}$ ), were evaluated. Control antibody staining is indicated by the dashed line, staining in control Ig-treated mice by the black line, and staining in anti-CD154-treated mice by the gray line. Data are representative of three separate experiments.

\section{Figure 4}

Frequencies of PLP139-151-specific Th1, Th2, and Th0 cells are comparable 100 days after immunization in control- and anti-CD154-treated mice. Splenic and lymph node CD4 ${ }^{+} \mathrm{T}$ cells were taken 100 days after immunization, and ELISPOT was used to determine the frequencies of Th1 (IFN- $\gamma$-secreting) cells, Th2 (IL-5-secreting) cells, and Th0 (IL-2-secreting) cells from lymph node and splenic compartments of control- and antiCD154-treated mice. Data, shown as mean \pm SEM, were obtained from three pooled samples and represent two separate experiments.

therapeutic potential in many models of autoimmunity $(6-8,10-12,17,22,27,31,32)$, transplantation (33-35, 35-37), and arteriosclerosis $(38,39)$. In EAE, blockade or elimination of the CD154-CD40 ligand pair interaction has been shown not only to prevent induction $(6-8,27)$, but, more importantly, to inhibit relapses when used for treatment of ongoing disease (8). Ligation of CD40 in the CNS by $\mathrm{CD} 154$ on infiltrating encephalitogenic $T$ cells has also recently been shown to be essential for the induction of optimal $\mathrm{T}$ cell effector function within the target organ $(15,16)$. This conclusion is supported by the observations that induction of CD 40 or CD40L expression on solid tumor cells $(40,41)$ or on skin keratinocytes (42) results in heightened $\mathrm{T}$ cell responses. Thus, CD154 blockade may primarily act to prevent $\mathrm{T}$ cell engagement in the CNS and subsequent recruitment of inflammatory cells, rather than affecting Th1 cells once they have become activated within the CNS.

We have previously reported that CD154 blockade concomitant with myelin peptide priming does not result in the preferential induction of specific Th2 versus Th1 cells in response to either PLP139-151 or the non-self OVA323-339 peptide (16), as had been proposed to be the mechanism of inhibition $(27,43)$. The numbers of Th 1 cells produced at the peak of the immune response (days 10-12 after immunization) were $40-50 \%$ lower in anti-CD154- than in control Ig-treated mice (16). However, at day 100 after
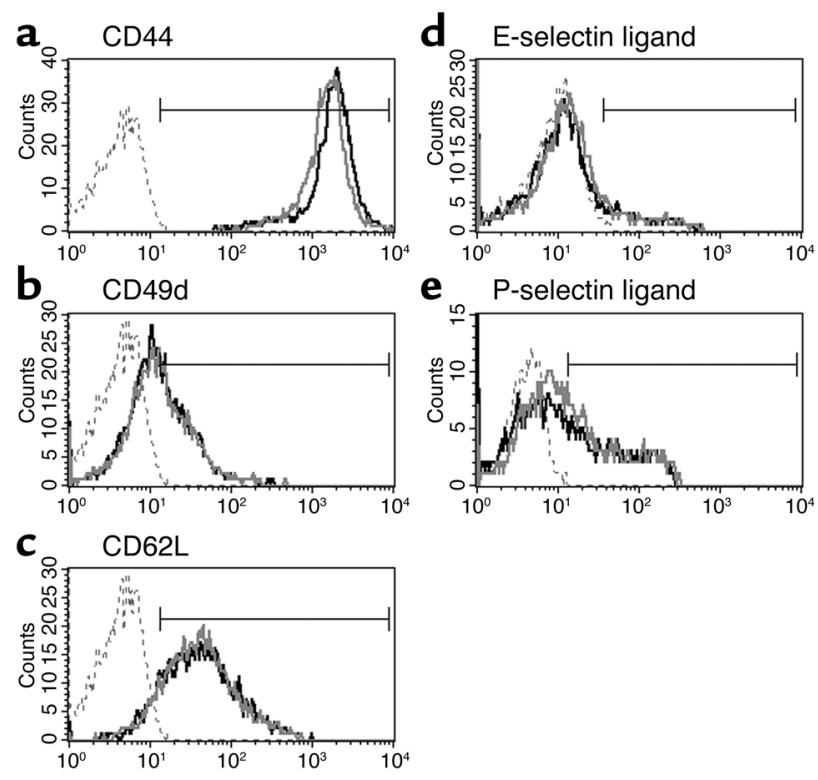


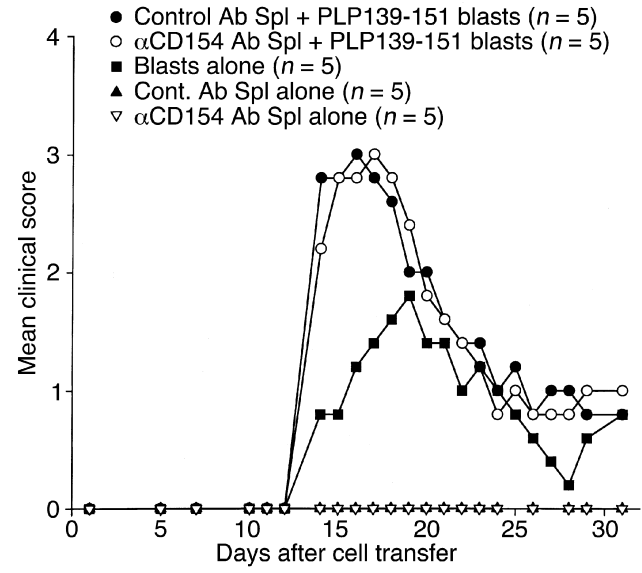

Figure 6

Peripheral $\mathrm{T}$ cells from asymptomatic anti-CD154-treated mice demonstrate full encephalitogenic capacity. Splenic (Spl) T cells were isolated from anti-CD154- and control Ig-treated mice 30 days after PLP139-151/CFA immunization. These cells $\left(5 \times 10^{6}\right.$ per recipient $)$ were then transferred to naive recipient mice with or without cotransfer of $2 \times 10^{6}$ encephalitogenic Th1 blasts. The Th1 blasts were obtained from the draining lymph nodes of mice 10 days after PLP139-151/CFA immunization and 4 days of in vitro reactivation in the presence of PLP139-151 peptide. Recipient mice were observed for clinical signs of disease for 31 days after transfer. Data are representative of two separate experiments with identical results.

immunization (Figure 4), there is no significant difference in the frequencies of peptide-specific Th 1 or Th2 cells, confirming that the that long-term Th1/Th2 balance is largely unaffected by CD154 blockade. It is possible that the reduced amplitude in acute-phase maximal response to encephalitogenic antigen plays a central role in determining the effectiveness of CD154 blockade in inhibiting Th1 effector function within the CNS. This argument is supported by the fact that, at the dosages used, a delay in treatment until later in disease is less effective (7). These observations raise a number of important questions regarding CD154-directed immunotherapy. First, given that a significant Th1 response is induced in the presence of anti-CD154 blockade, what is the long-term efficacy of CD154 blockade at inhibiting the induction of clinical autoimmune disease? Second, is Th1 differentiation functionally different under the influence of CD154 blockade? Most importantly, can autoantigen-specific Th1 cells that differentiated in the presence of anti-CD154 treatment later contribute to clinical disease?

We show that short-term anti-CD154 treatment of mice at the time of immunization results in long-term inhibition of disease (Figure 1). While 2 of 16 mice $(12.5 \%)$ did break through, disease onset was significantly delayed and the mice demonstrated very mild clinical disease. In contrast to its long-term therapeutic efficacy, anti-CD154 therapy does not have a longterm inhibitory influence on Th1 development. One hundred days after immunization, anti-CD154-treated mice did not show any significant difference in fre- quency and showed only mild reduction in IFN- $\gamma$ secretion in vitro compared with controls. However, Th1 cells appear less effective in mediating DTH responses, even 100 days after immunization when the antiCD154 antibody has been cleared from the mouse. Thus, while Th1 cells appear to be functional in vitro, they demonstrate a functional deficit in vivo that cannot be simply explained by fewer circulating cells at this time point. In this regard, there are some important differences between $T$ cell activation in animals treated with anti-CD154 and in CD40 knockout mice. CD40 knockout mice do not display a sustained immune response to immunizing antigens (17-20). This is in direct contrast to anti-CD154-treated mice, in which ongoing immune responses can be seen even 100 days after immunization (Figures 2-4) (16). Although we show that CD154 blockade almost completely ablated PLP139-151-specific B cell responses 25 days after immunization (Figure 2a), based on the maintenance of antigen-specific responses of lymph node $\mathrm{T}$ cells, there appears to be little long-term effect on dendritic cell function, compared with the dramatic dendritic cell deficit observed in CD40 knockout mice (17). We are currently addressing the possibility that high levels of CD40 engagement results in the classical Th1 skewing through induction of IL-12 (14), while only low levels of CD40 engagement may be needed for APC survival and maintenance of the immune response.

We also show that antigen-specific $T$ cells from antiCD154-treated mice have normal percentages and expression levels of two homing receptors relevant to entry into the CNS (CD44 and CD49d) as well as other homing receptors (P-selectin ligand and E-selectin ligand), the latter two being critically dependent upon IL-12 for their expression (44-46). We are currently examining whether CD154 blockade affects APC production of IL-12 and under what conditions IL-12 can be inhibited, since IL-12 may be induced in a CD40dependent or -independent manner $(23,47)$.

Most interestingly, we show (Figure 6) that peripheral PLP139-151-specific $T$ cells in asymptomatic antiCD154-treated mice retained their encephalitogenic capacity. This was demonstrated by their ability to enhance clinical disease severity when cotransferred to naive recipients with suboptimal numbers of PLP139-151-specific in vitro-activated $T$ cell blasts. Thus, $\mathrm{T}$ cells primed in the presence of $\mathrm{CD} 154$ blockade display in vitro and in vivo functional capabilities comparable to those of $\mathrm{T}$ cells from control Ig-treated mice and continue to persist for at least 100 days following cessation of the antibody treatment. The fact that shortterm CD154 blockade results in long-term inhibition of clinical disease suggests that these persistant and circulating myelin-specific Th1 cells do not cause disease. Indeed, we found that transfer of splenic $\mathrm{T}$ cells from MR-1-treated mice alone does not result in clinical disease, but that they contribute to disease only when given in conjunction with in vitro-activated encephalitogenic $\mathrm{T}$ cells. The transferred cells must therefore be recruit- 
ed to the CNS by lymph node-derived, in vitro-reactivated, encephalitogenic cells. Since these cells do not readily induce clinical disease, either upon adoptive transfer or in the immunized MR-1-treated donor animal, the long-term effectiveness of MR-1 therapy may therefore lie in its ability to prevent the initial effector function and recruitment of host inflammatory cells within the CNS through blockade of chemokine secretion and APC engagement $(15,16)$. Once this diseaseinitiating period has passed, the remaining peripheral cells are no longer capable of initiating disease. Our data suggest that such disease-provoking cells are present only transiently in the initial wave of the immune response. This argument is further supported by the fact that CD154 blockade is only transiently effective in inhibiting disease, in that mice immunized with encephalitogenic antigen, treated with MR-1, and then later reimmunized develop acute EAE (48). Alternately, it is possible that CD154 blockade promotes the activation of a regulatory population that prevents the encephalitogenic action of the peripherally antigen-specific cells, which might explain the continued reduction observed in DTH responses to the immunizing antigen. We are currently addressing this hypothesis.

These data raise a potentially important caution when considering the immunotherapeutic potential of anti-CD154 treatment. For MS, in which disease induction and clinical relapses have been associated with virus infections (3), anti-CD154 antibody therapy may only be effective in treating forms of disease not associated with a persistent or latent CNS infection that could lead to renewed inflammatory and cellular recruitment signals. However, where it may be effective is in a therapeutic setting in which disease may have originally been caused via molecular mimicry or bystander activation during a transient viral infection either in the CNS or in the periphery (5), but that viral presence is not persistent.

Collectively, our current and previous $(8,16)$ results indicate that although frequency and function of Th1 cells are delayed and reduced in the short term by antiCD154 treatment, peripheral immune responses eventually develop at significant levels and are maintained over a long period of time. The data suggest that during the induction of the autoimmune response, $\mathrm{T}$ cells are produced that can initiate an immune reaction in the CNS, but that this is a transient event that must occur during a specified time interval. We further show that $\mathrm{T}$ cells induced under the cover of CD154 blockade are capable of contributing to clinical severity, but not initiation of clinical disease. That these cells remain circulating and are maintained for such a long time without causing clinical disease suggests that they may contribute only when another initiator cell type is induced. This has potentially important clinical consequences in recurrent or chronic autoimmune disease. These peripheral cells may potentially contribute to the increased clinical disease that accompanies epitope spreading whereby the initial epitope-specific $T$ cells are activated and cause clinical disease, releasing new antigens to the immune system. The $T$ cells specific for the initial epitope may not be central to the initiation of relapse but may contribute to clinical disease initiated by $\mathrm{T}$ cells activated to endogenous antigens. It is also possible that these $T$ cells could be reactivated by innate immune signals induced by a subsequent infection. This suggests that a prudent direction for immune intervention with CD154 blockade would be to combine this treatment with additional immunosuppressive therapies to prevent their possible reexpression of critical homing molecules and/or reactivation of their encephalitogenic effector functions.

\section{Acknowledgments}

This work was supported by US Public Health Service NIH Research grants NS-34819, NS-30871, and U19 AI/DK-51973, and National Multiple Sclerosis Society grant RG2275.

1. Brown, A.M., and McFarlin, D.E. 1981. Relapsing experimental allergic encephalomyelitis in the SJL/J mouse. Lab. Invest. 45:278-284.

2. McRae, B.L., Kennedy, M.K., Tan, L.J., Dal Canto, M.C., and Miller, S.D. 1992. Induction of active and adoptive chronic-relapsing experimental autoimmune encephalomyelitis (EAE) using an encephalitogenic epitope of proteolipid protein. J. Neuroimmunol. 38:229-240.

3. Kurtzke, J.F. 1993. Epidemiologic evidence for multiple sclerosis as an infection. Clin. Microbiol. Rev. 6:382-427.

4. Waksman, B.H. 1995. Multiple sclerosis: more genes versus environment. Nature. 377:105-106.

5. Olson, J.K., Croxford, J.L., and Miller, S.D. 2001. Virus-induced autoimmunity: potential role of viruses in initiation, perpetuation, and progression of $\mathrm{T}$ cell-mediated autoimmune diseases. Viral Immunol. 14:227-250.

6. Grewal, I.S., et al. 1996. Requirement for CD40 ligand in costimulation induction, T cell activation, and experimental allergic encephalomyelitis. Science. 273:1864-1867.

7. Gerritse, K., et al. 1996. CD40-CD40 ligand interactions in experimental allergic encephalomyelitis and multiple sclerosis. Proc. Natl. Acad. Sci. USA. 93:2499-2504.

8. Howard, L.M., et al. 1999. Mechanisms of immunotherapeutic intervention by anti-CD40L (CD154) antibody in an animal model of multiple sclerosis. J. Clin. Invest. 103:281-290.

9. Balasa, B., et al. 1997. CD40 ligand-CD40 interactions are necessary for the initiation of insulitis and diabetes in nonobese diabetic mice. $J$. Immunol. 159:4620-4627.

10. Durie, F.H., et al. 1993. Prevention of collagen-induced arthritis with an antibody to gp39, the ligand for CD40. Science. 261:1328-1330.

11. Carayanniotis, G., Masters, S.R., and Noelle, R.J. 1997. Suppression of murine thyroiditis via blockade of the CD40-CD40L interaction. Immunology. 90:421-426.

12. Griggs, N.D., et al. 1996. The relative contribution of the CD28 and gp39 costimulatory pathways in the clonal expansion and pathogenic acquisition of self-reactive T cells. J. Exp. Med. 183:801-810.

13. Stout, R.D., and Suttles, J. 1996. The many roles of CD40 in cell-mediated inflammatory responses. Immunol. Today. 17:487-492.

14. Van Kooten, C., and Banchereau, J. 1997. Functional role of CD40 and its ligand. Int. Arch. Allergy Immunol. 113:393-399.

15. Becher, B., Durell, B.G., Miga, A.V., Hickey, W.F., and Noelle, R.J. 2001. The clinical course of experimental autoimmune encephalomyelitis and inflammation is controlled by the expression of CD40 within the central nervous system. J. Exp. Med. 193:967-974.

16. Howard, L.M., and Miller, S.D. 2001. Autoimmune intervention by CD154 blockade prevents $\mathrm{T}$ cell retention and effector function in the target organ. J. Immunol. 166:1547-1553.

17. Namba, K., et al. 2000. Amelioration of experimental autoimmune uveoretinitis by pretreatment with a pathogenic peptide in liposome and anti-CD40 ligand monoclonal antibody. J. Immunol. 165:2962-2969.

18. Grewal, I.S., and Flavell, R.A. 1996. A central role of CD40 ligand in the regulation of CD4+ T-cell responses. Immunol. Today. 17:410-414.

19. Van Kooten, C., and Banchereau, J. 1996. CD40-CD40 ligand: a multifunctional receptor-ligand pair. Adv. Immunol. 61:1-77.

20. Caux, C., et al. 1994. Activation of human dendritic cells through CD40 cross-linking. J. Exp. Med. 180:1263-1272. 
21. Grewal, I.S., and Flavell, R.A. 1998. CD40 and CD154 in cell-mediated immunity. Annu. Rev. Immunol. 16:111-135.

22. Stuber, E., Strober, W., and Neurath, M. 1996. Blocking the CD40L-CD40 interaction in vivo specifically prevents the priming of T helper 1 cells through the inhibition of interleukin 12 secretion. J. Exp. Med. 183:693-698.

23. DeKruyff, R.H., Gieni, R.S., and Umetsu, D.T. 1997. Antigen-driven but not lipopolysaccharide-driven IL-12 production in macrophages requires triggering of CD40. J. Immunol. 158:359-366.

24. Nieland, J.D., Graus, Y.F., Dortmans, Y.E., Kremers, B.L.J.M., and Kruisbeek, A.M. 1998. CD40 and CD70 co-stimulate a potent in vivo antitumor $\mathrm{T}$ cell response. J. Immunother. 21:225-236.

25. Dal Canto, M.C., and Lipton, H.L. 1975. Primary demyelination in Theiler's virus infection. An ultrastructural study. Lab. Invest. 33:626-637.

26. Laman, J.D., et al. 1998. Therapy with antibodies against CD40L (CD154) and CD44-variant isoforms reduces experimental autoimmune encephalomyelitis induced by a proteolipid protein peptide. Mult. Scler 4:147-153.

27. Samoilova, E.B., Horton, J.L., Zhang, H.D., and Chen, Y.H. 1997. CD40L blockade prevents autoimmune encephalomyelitis and hampers TH1 but not TH2 pathway of T cell differentiation. J. Mol. Med. 75:603-608.

28. Durandy, A., et al. 1993. Induction by anti-CD40 antibody or soluble CD40 ligand and cytokines of IgG, IgA and IgE production by B cells from patients with X-linked hyper IgM syndrome. Eur. J. Immunol. 23:2294-2299.

29. Banchereau, J., et al. 1994. The CD40 antigen and its ligand. Annu. Rev. Immunol. 12:881-922.

30. Lei, X.F., et al. 1998. Disruption of antigen-induced inflammatory responses in CD40 ligand knockout mice. J. Clin. Invest 101:1342-1353.

31. Mohan, C., Shi, Y., Laman, J.D., and Datta, S.K. 1995. Interaction between CD40 and its ligand gp39 in the development of murine lupus nephritis. J. Immunol. 154:1470-1480.

32. Early, G.S., Zhao, W., and Burns, C.M. 1996. Anti-CD40 ligand antibody treatment prevents the development of lupus-like nephritis in a subset of New Zealand black x New Zealand white mice. Response correlates with the absence of an anti-antibody response. J. Immunol. 157:3159-3164.

33. Blazar, B.R., et al. 1997. Blockade of CD40 ligand-CD40 interaction impairs CD4+ T cell-mediated alloreactivity by inhibiting mature donor $\mathrm{T}$ cell expansion and function after bone marrow transplantation. $J$. Immunol. 158:29-39.

34. Funakoshi, S., et al. 1997. Immunologic and hematopoietic effects of CD40 stimulation after syngeneic bone marrow transplantation in mice. J. Clin. Invest. 99:484-491.
35. Larsen, C.P., et al. 1996. CD40-gp39 interactions play a critical role during allograft rejection. Suppression of allograft rejection by blockade of the CD40-gp39 pathway. Transplantation. 61:4-9.

36. Larsen, C.P., et al. 1996. Long-term acceptance of skin and cardiac allografts after blocking CD40 and CD28 pathways. Nature. 381:434-438.

37. Graca, L., Honey, K., Adams, E., Cobbold, S.P., and Waldmann, H. 2000. Cutting edge: anti-CD154 therapeutic antibodies induce infectious transplantation tolerance. J. Immunol. 165:4783-4786.

38. Mach, F., Schonbeck, U., Sukhova, G.K., Atkinson, E., and Libby, P. 1998. Reduction of atherosclerosis in mice by inhibition of CD40 signalling. Nature. 394:200-203.

39. Lamans, J.D., de Smet, B.J., Schoneveld, A., and Van Meurs, M. 1997. CD40-CD 40L interactions in atherosclerosis. Immunol. Today. 18:272-277.

40. Alexandroff, A.B., et al. 2000. Role for CD40-CD40 ligand interactions in the immune response to solid tumours. Mol. Immunol. 37:515-526.

41. Sun, Y., et al. 2000. In vivo gene transfer of CD40 ligand into colon cancer cells induces local production of cytokines and chemokines, tumor eradication and protective antitumor immunity. Gene Ther. 7:1467-1476.

42. Mehling, A., et al. 2001. Overexpression of CD40 ligand in murine epidermis results in chronic skin inflammation and systemic autoimmunity. J. Exp. Med. 194:615-628.

43. Howland, K.C., Ausubel, L.J., London, C.A., and Abbas, A.K. 2000. The roles of CD28 and CD40 ligand in T cell activation and tolerance. $J$. Immunol. 164:4465-4470

44. Wagers, A.J., Waters, C.M., Stoolman, L.M., and Kansas, G.S. 1998. Interleukin 12 and interleukin 4 control $\mathrm{T}$ cell adhesion to endothelial selectins through opposite effects on alpha1, 3-fucosyltransferase VII gene expression. J. Exp. Med. 188:2225-2231.

45. Kansas, G.S., and Tedder, T.F. 1991. Transmembrane signals generated through MHC class II, CD19, CD20, CD39, and CD40 antigens induce LFA-1-dependent and independent adhesion in human B cells through a tyrosine kinase-dependent pathway. J. Immunol. 147:4094-4102.

46. Hollenbaugh, D., et al. 1995. Expression of functional CD40 by vascular endothelial cells. J. Exp. Med. 182:33-40.

47. Kato, T., Hakamada, R., Yamane, H., and Nariuchi, H. 1996. Induction of IL-12 p40 messenger RNA expression and IL-12 production of macrophages via CD40-CD40 ligand interaction. J. Immunol. 156:3932-3938.

48. Girvin, A.M., Dal Canto, M.C., and Miller, S.D. 2002. CD40/CD40L interaction is essential for the induction of EAE in the absence of CD28mediated costimulation. J. Autoimmunol. In press. 